\title{
ARTICLE
}

\section{Effect of the aldose reductase inhibitor fidarestat on experimental diabetic neuropathy in the rat}

\author{
Y. Kuzumoto • S. Kusunoki • N. Kato • M. Kihara • \\ P. A. Low
}

Received: 24 December 2005 / Accepted: 30 June 2006 / Published online: 25 October 2006

(C) Springer-Verlag 2006

\begin{abstract}
Aims/hypothesis Fidarestat, an aldose reductase inhibitor (ARI), has been reported to improve clinical symptoms and nerve conduction deficits in human diabetic neuropathy. We evaluated the dose-dependency and some of the mechanisms of the drug action in experimental diabetic neuropathy (EDN). Methods Control rats and rats with EDN were fed on normal pellets or pellets containing $0.00066 \%(1 \mathrm{mg} / \mathrm{kg}$ ) or $0.00263 \%$ $(4 \mathrm{mg} / \mathrm{kg}$ ) fidarestat for 10 weeks. We evaluated the effect of fidarestat on nerve blood flow (NBF), electrophysiology, and sorbitol and fructose content in sciatic nerve in control and diabetic rats. For detection of oxidative stress in peripheral nerve, we measured sciatic nerve reduced glutathione (GSH) and 8-hydroxy-2'-deoxyguanosine (8-OHdG) immunolabelling of dorsal root ganglion (DRG) neurons.

Results NBF, compound muscle action potential and amplitude of C-potential were significantly improved in diabetic rats fed on the diet supplemented with fidarestat. Fidarestat suppressed the increase in sorbitol and fructose,
\end{abstract}

Y. Kuzumoto $(\varangle) \cdot$ S. Kusunoki

Department of Neurology, Kinki University, School of Medicine,

377-2 Ohno-higashi Osaka-Sayama,

Osaka, Japan

e-mail: kuzumoto@neuron.med.kindai.ac.jp

\section{N. Kato}

Pharmaceutical Research Laboratories,

Sanwa Kagaku Kenkyusho Company,

363 Shiosaki Hokusei, Inabe,

Mie, Japan

M. Kihara

Department of Neurology, Kawamura Hospital,

1-84 Akutamidaihanya Gifu,

Gifu, Japan

P. A. Low

Department of Neurology, Mayo Clinic,

Rochester, MN, USA normalised GSH in sciatic nerve, and reduced the number of 8-OHdG-positive cells in DRG.

Conclusions/interpretation Fidarestat improves neuropathy, presumably via an improvement in oxidative stress. This study supports a role for fidarestat in the treatment of diabetic neuropathy.

\section{Keywords}

8-Hydroxy-2'-deoxyguanosine - Diabetic neuropathy ·

Dorsal root ganglion - Fidarestat $\cdot$ Fructose $\cdot$ Glutathione .

Nerve blood flow $\cdot$ Nitric monoxide $\cdot$ Sciatic nerve $\cdot$ Sorbitol

\begin{tabular}{|c|c|}
\hline \multicolumn{2}{|c|}{ Abbreviations } \\
\hline ARI & aldose reductase inhibitor \\
\hline CMAP & compound muscle action velocity \\
\hline DRG & dorsal root ganglion \\
\hline EDN & experimental diabetic neuropathy \\
\hline 8-OHdG & 8-hydroxy-2'-deoxyguanosine \\
\hline GSH & reduced glutathione \\
\hline MAPK & mitogen-activated protein kinase \\
\hline MAP & mean arterial blood pressure \\
\hline $\mathrm{MCV}$ & motor conduction velocity \\
\hline $\mathrm{NBF}$ & nerve blood flow \\
\hline $\mathrm{NCV}$ & nerve conduction velocity \\
\hline $\mathrm{NO}$ & nitric oxide \\
\hline NOLA & $N^{\omega}$-nitro-L-arginine \\
\hline NOS & nitric oxide synthase \\
\hline STZ & streptozotocin \\
\hline
\end{tabular}

\section{Introduction}

Chronic hyperglycaemia results in human and experimental diabetic neuropathy (EDN), with slowing of nerve conduction. A number of key alterations have been described. Polyol 
pathway hyperactivity [1], myoinositol reduction [2, 3], reduction in $\mathrm{Na}^{+}, \mathrm{K}^{+}$-ATPase activity [2, 4], reduction in nerve blood flow (NBF) and oxygenation [5-9], reduction in nitric oxide (NO) or NO synthase (NOS) $[5,10,11]$ and evidence of increased oxidative stress [11, 12]. Recent work from several research centres has led to a more integrated and unified hypothesis on the pathogenesis of diabetic neuropathy $[11,12]$. A key link between hyperglycaemia and neuropathy is that of aldose reductase hyperactivity [13, 14]. The aldose reductase inhibitor (ARI), fidarestat, administered in the short term $[15,16]$ or for a longer duration [17], reduces polyol pathway activity and improves neuropathy.

The aims of this study were to examine whether fidarestat could correct nerve conduction deficit and to examine some possible mechanisms, including nerve perfusion deficit, NO status and oxidative stress-related damage in diabetic neuropathy.

\section{Materials and methods}

Animals

We used male Sprague-Dawley rats with initial body weights of $\sim 250 \mathrm{~g}$. The animals were housed in cages and received an unrestricted supply of water and rat chow (CE2; CLEA, Inc., Tokyo, Japan).

Experimental diabetes was induced in 22 rats using streptozotocin (STZ), administered by i.v. injection. STZ was dissolved in $0.05 \mathrm{~mol} / \mathrm{l}$ sodium citrate at $\mathrm{pH} 4.5$ $(20 \mathrm{mg} / \mathrm{ml}$, dose $=1.00 \mathrm{ml} / \mathrm{kg})$. Age-matched control rats were injected with sodium citrate. Rats were presumed diabetic if their fasting blood glucose level exceeded $16.7 \mathrm{mmol} / \mathrm{l} 2$ weeks after injection of STZ and remained greater than $16.7 \mathrm{mmol} / \mathrm{l}$ throughout the study.

Fidarestat supplementation study

We separated 37 rats into five groups. After 2 weeks of diabetes one group of rats was started on a diet of treated pellets for 10 weeks while the other diabetic group and the aged-matched control group continued to receive a normal diet ( $\mathrm{N}$ : control rats receiving normal diet, $n=7 ; \mathrm{N}+4$ : control rats receiving fidarestat $4 \mathrm{mg} / \mathrm{kg}$ diet, $n=8 ; \mathrm{D}$ : diabetic rats receiving normal diet, $n=9 ; \mathrm{D}+1$ : diabetic rats receiving fridarestat $1 \mathrm{mg} / \mathrm{kg}$ diet for 10 weeks, $n=6$; $\mathrm{D}+4$ : diabetic rats receiving fidarestat $4 \mathrm{mg} / \mathrm{kg}$ diet for 10 weeks, $n=7$ ).

\section{Nerve preparation}

The details of the nerve preparation have been described previously $[5,18]$. In brief, the rats were anaesthetised with an i.p. injection of pentobarbital sodium $(100 \mathrm{mg} / \mathrm{kg})$ that induced general anaesthesia in rats for $2-3 \mathrm{~h}$. Rectal temperature was monitored and maintained between 36.5 and $37.5^{\circ} \mathrm{C}$ using a thermister probe connected to a digital thermometer attached to a control feedback unit (BWT-100, Bio Research Center Company, Nagoya, Japan) and an infrared lamp. A tracheotomy tube was inserted and a polyethylene catheter (PE-50) was placed into the left common carotid artery for monitoring mean arterial blood pressure (MAP) and for measurement of blood gas and $\mathrm{pH}$. Artificial ventilation with a mixture of nitrogen and oxygen was provided by a rodent respirator (Model 683, Harvard Apparatus, Mills, MA, USA). The animals were paralysed with tubocurarine (15-20 U/kg, intra-arterial or i.p.), which maintains muscle relaxation for 3-4 h. The MAP was measured with a pressure transducer (ASI202, NEC, Tokyo, Japan) and recorded on a polygraph chart recorder (Model R202, Rikadenki, Tokyo, Japan). Depth of anaesthesia was assessed by continuous monitoring of MAP, which showed no spontaneous fluctuations and no change in response to manipulation of tissue, under sufficiently deep anaesthesia. When such fluctuations appeared, intra-arterial bolus injections of $8-12 \mathrm{mg} / \mathrm{kg}$ pentobarbital were given. When necessary, additional intra-arterial doses of 3-5 U/kg tubocurarine were given slowly to abolish muscle twitches. Arterial blood was sampled every $60 \mathrm{~min}$ to ensure that $\mathrm{pH}$ and gas values remained within the physiological range. A polyethylene tube ( $3 \mathrm{~mm}$ diameter) filled with $2 \mathrm{mmol} / \mathrm{K} \mathrm{KCl}$ and 3\% agar was sutured into the subcutaneous tissue of the abdominal wall. The other end of this tube was placed in a $2 \mathrm{mmol} / \mathrm{KCl}$ solution into which a silver chloride electrode connected to the reference terminal of a current-sensitive amplifier (Chemical Microsensor Model 1201, Diamond Electro Tech, Ann Arbor, MI, USA) was also placed. A length of left sciatic nerve, not exceeding $2 \mathrm{~cm}$, was exposed and a pool was formed with the surrounding muscle and skin. The pool was filled with physiological saline $(154 \mathrm{mmol} / 1 \mathrm{NaCl})$ maintained at $33.5 \pm 0.1^{\circ} \mathrm{C}$ using a servo-controlled infrared lamp.

\section{Measurements of NBF}

$\mathrm{NBF}$ was measured by microelectrode $\mathrm{H}_{2}$-polarography (tip size $2-5 \mu \mathrm{m})$. The details of the procedure have been described previously $[5,18]$. In brief, the hydrogensensitive microelectrode, which demonstrated a linear response to varying concentrations of hydrogen in saline, was inserted into the endoneurium. The free end of the electrode was connected to the current-sensitive amplifier and was polarised positively with $+0.25 \mathrm{~V}$. The tissue was then saturated with hydrogen to reach a plateau (indicating hydrogen saturation in endoneurium), then the hydrogen supply was shut off and a hydrogen clearance curve was 
recorded on a polygraph chart recorder for more than $30 \mathrm{~min}$, until the current reached baseline. The signal was fed into a computer (PC-XT) and was stored (Lotus 123, Lotus Development, MA, USA). The hydrogen clearance curves obtained were fitted to a mono- or biexponential washout curve using the non-linear regression analysis program based on the Levenberg-Marquardt algorithm.

\section{Electrophysiological methods}

The electrophysiological studies were performed using techniques that are standard for our laboratory [19]. In brief, motor conduction velocities (MCVs) and the amplitude of the compound muscle action potential were measured in the sciatic-tibial nerves. Fine stainless-steel near-nerve stimulating and recording electrodes were employed. The compound muscle action potential (CMAP) was recorded from the dorsum of the hind paw while the nerve was stimulated at the level of the sciatic notch and ankle. We measured CMAP amplitude and latency to onset after proximal and distal stimulation. The $\mathrm{MCV}$ was calculated. Recordings were made at $35^{\circ} \mathrm{C}$.

Effects of epineurially applied NOS inhibitor

The left sciatic nerve was exposed, and a pool was formed with surrounding muscle and skin and filled with mammalian Ringer solution. Once the resting NBF was measured we replaced the mammalian Ringer solution with a $N^{\omega}$. nitro-L-arginine (NOLA) solution (concentration of $10^{-3} \mathrm{mmol} / 1$ in mammalian Ringer solution) and bathed the nerve for $30 \mathrm{~min}$. After $30 \mathrm{~min}$ of NOLA application we measured NBF again.

In vitro nerve recordings

The following experiments were performed according to previously described methodology $[8,10,20]$. The rats were anaesthetised with an i.p. injection of pentobarbital sodium $(60 \mathrm{mg} / \mathrm{kg})$. The left sciatic nerve was resected and immediately placed in Ringer's solution. Within a few minutes, blood vessels, fat and epineurium were dissected away from the fascicles under a dissecting microscope. The nerve was placed directly onto 16 parallel, $0.5 \mathrm{~mm}$ diameter, platinum electrodes $(5 \mathrm{~mm}$ interelectrode distance) in a Perspex chamber bathed in Ringer's solution, maintained at $33.5^{\circ} \mathrm{C}$ with a servo-controlled infrared lamp. The stimulus was applied through the electrode at the rostral end of the nerve, and the action potential recorded along different recording electrodes toward the caudal end the nerve. The response was amplified $\times 1000$, stored on a computer and analysed using Nicolet TN5200 (Texas Instruments, Madison, WI, USA).
Measurements were recorded immediately upon placing nerve in the chamber. The action potential was amplified using solid state, low-noise differential amplifiers. The conduction velocity of the fastest fibres of each component of the compound action potential was calculated by dividing the distance between the stimulating cathode and the active recording electrode by the latency measured from onset of the stimulus artefact to the onset of each negative component of the compound action potential. The amplitude of the action potential was measured from baseline to peak.

\section{Determination of sorbitol and fructose contents in nerve}

The sciatic nerve was homogenised in distilled water. TCA and internal standard (D-sorbitol and D-fructose) were added to the homogenate, followed by centrifugation at $10,000 \mathrm{~g}$ for $5 \mathrm{~min}$ at $4^{\circ} \mathrm{C}$ to obtain the supernatant fraction. To remove TCA, the supernatant fraction was washed with ethyl ether. Sorbitol and fructose in the supernatant fraction were converted to sorbitol acetate derivative and fructose acetate derivative according to the method described by Gurrant and Moss [21] (HPLC with the HP1050; Hewlett Packard, Palo Alto, CA, USA) and mass spectrometry (TSQ; Finnigan Mat, San Jose, CA, USA) using a Cadenza CD-C18 column $(75 \times 2.0 \mathrm{~mm}$, internal diameter $3 \mathrm{~mm}$; Imtakt, Kyoto, Japan).

\section{Determination of GSH in nerve}

The frozen sciatic nerve was pulverised in a cooled glass mortar after the addition of ice-cold 5\% metaphosphoric acid. The tissue homogenates were centrifuged at $3,000 \mathrm{~g}$ for $10 \mathrm{~min}$ at $4^{\circ} \mathrm{C}$. Aliquots of the supernatant fractions were then used for determination of glutathione (GSH) with colorimetric assay kit (Bioxytech GSH-400) supplied by Oxis Research (Portland, OR, USA).

Immunohistochemistry for 8-hydroxy-2'-deoxyguanosine

The immunohistochemical procedure was performed using previously described methods with modifications described below $[22,23]$. Dorsal root ganglion (DRG) (L5) was fixed in Bouin's solution and embedded in paraffin. Immunostaining was conducted on the de-paraffinised transverse sections of DRG. After de-paraffinisation, the sections were treated with $3 \% \mathrm{H}_{2} \mathrm{O}_{2}$ in methanol for 10 min to inactivate endogenous peroxidase, $0.05 \mathrm{mmol} / 1 \mathrm{NaOH}$ in $40 \%$ ethanol for $12 \mathrm{~min}$ to denature DNA, $250 \mu \mathrm{g} / \mathrm{ml}$ RNase for $1 \mathrm{~h}$ at $37^{\circ} \mathrm{C}$, and then incubated in $10 \%$ horse serum in Trisbuffered saline for $1 \mathrm{~h}$. The sections were next incubated with diluted 8-hydroxy-2'-deoxyguanosine (8-OHdG) antibody (1:100, Nikken Foods Co., Shizuoka, Japan) at $4^{\circ} \mathrm{C}$ overnight, and then sequentially exposed to biotinylated anti-mouse IgG and avidin-biotin complex (Vectastain 
ABC Elite kit, Vector Laboratories, Burlingame, CA, USA). The sites of peroxidase binding were demonstrated with 3-amino-9-ethyl carbazole.

The 8-OHdG-positive neurons were defined as the neurons with red reaction products in the nucleus, the intensity of which was more than two times that of background staining (mean cytoplasmic intensity in DRG neuron of normal rat). The intensity was determined using images with a final magnification of $\times 200$ with the aid of a colour image analyser (IPAP-WIN, Sumika Technoservice, Osaka, Japan) connected to a camera control unit (XC-003, Sony, Tokyo, Japan). Prevalence of positive neurons was calculated with a mean of 110 nuclei in each rat.

\section{Measurement of the diameter of DRG neuron}

The diameter of DRG neuron was measured with the aid of a colour image analyser system as well as $8-\mathrm{OHdG}$ analyses. Neuron size was defined as the area delineated by the outer cytoplasm, and the diameter was calculated. For determination of the diameter 154-191 neurons per DRG were used. After evaluation of the mean value of DRG diameter, we scanned each diameter histogram and divided the neurons into large $(>28 \mu \mathrm{m})$, medium $(20 \mu \mathrm{m}<$ diameter $<28 \mu \mathrm{m})$ and small $(<20 \mu \mathrm{m})$ according to the criteria of Kishi et al. [24].

The experiments involving animals were approved by the local ethics committee in Kinki University.

Statistical analysis

Statistical analysis was performed on a Macintosh computer system using the Stat View (ABACUS Concept, Berkeley, CA, USA) statistical program. The data were analysed using chi-squared, non-parametric comparison, analysis of variance (ANOVA) when comparing differences between the three groups. Data within two groups were analysed using the unpaired group $t$ test (Welch method) and Wilcoxon rank sum test. Values are expressed as mean \pm SEM and significance was accepted when $p<0.05$.

\section{Results}

Blood glucose and body weight

The blood glucose values in the three groups were (mean $\pm \mathrm{SE}$ ): $\mathrm{N}, 5.7 \pm 0.02 \mathrm{mmol} / \mathrm{l} ; \mathrm{N}+4,5.5 \pm 0.02 \mathrm{mmol} / \mathrm{l} ; \mathrm{D}, 27.3 \pm$ $0.03 \mathrm{mmol} / \mathrm{l} ; \mathrm{D}+1,27.7 \pm 0.04 \mathrm{mmol} / \mathrm{l} ;$ and $\mathrm{D}+4,27.5 \pm$ $0.04 \mathrm{mmol} / \mathrm{l}$. All of the diabetic groups had significantly higher blood glucose levels than the control group. Body weights at the time of killing were as follows: $\mathrm{N} 408.1 \pm 5.1 \mathrm{~g}, \mathrm{~N}+4430.0 \pm$

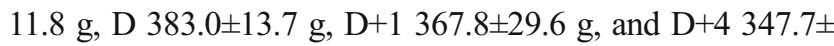
$9.8 \mathrm{~g}$.
$\mathrm{NBF}$

The D group had a significant reduction in NBF compared with the control group (D vs N, $p<0.001$ ) and resembled normal nerves treated with NOLA. Fidarestat supplementation dose-dependently and significantly improved NBF in diabetic rats (Fig. 1).

Electrophysiology

CMAP and MCV were significantly reduced in the diabetic animals $(p<0.001)$. In the $\mathrm{D}+1$ group, CMAP and MCV improved and approached statistical significance $(p=0.07)$. In the $\mathrm{D}+4$ group, both CMAP and the MCV were significantly increased with fidarestat diet (CMAP $p<0.05$, MCV $p<0.001$ ) (Fig. 2).

NOS inhibitor studies on diabetic nerves

NBF was significantly reduced in diabetic nerves (Fig. 1). Application of NOLA solution in the D group resulted in no further reduction. In contrast, in the $\mathrm{D}+1$ and $\mathrm{D}+4$ groups, NBF deficit was prevented and the reduction followed NOLA was restored, resembling the response in controls ( $\mathrm{N}$ and $\mathrm{N}+4$ groups). NOLA treatment had no effect in any group. NBF at baseline and after immersion in NOLA containing solution were: N 15.90 $\pm 1.1,9.8 \pm 0.8$; $\mathrm{N}+4$ 16.6 $\pm 1.2,10.8 \pm 0.8 ; \mathrm{D} 8.5 \pm 1.7,8.4 \pm 0.4 ; \mathrm{D}+115.7 \pm 2.1$, $10.4 \pm 1.0$; and D+4 18.9 $\pm 1.6,7.3 \pm 0.8$, respectively (Fig. 1).

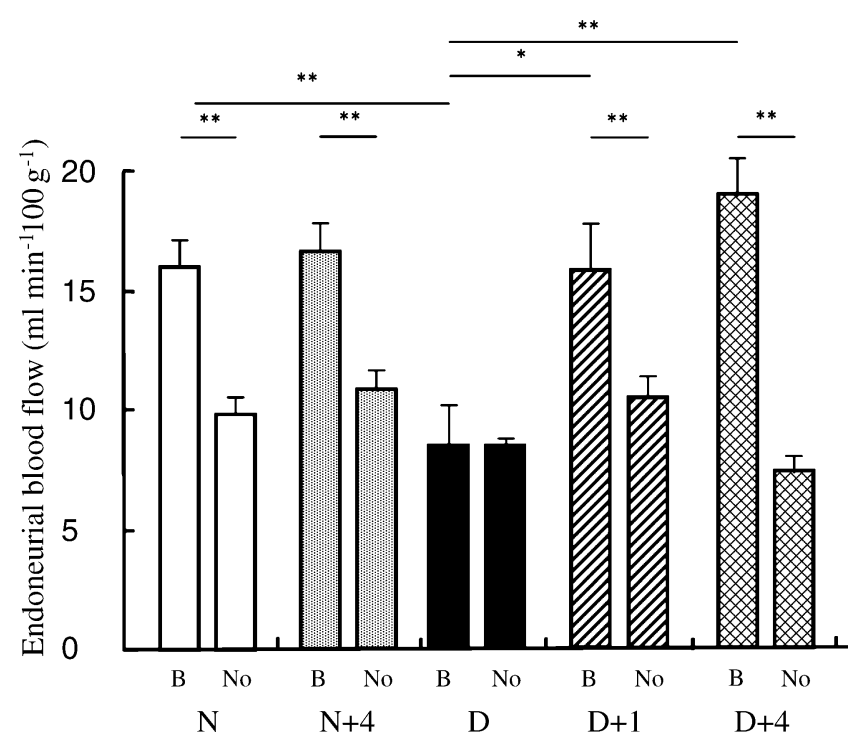

Fig. $1 \mathrm{NBF}$ at baseline (B; before application of NOLA) and after application of NOLA solution (No). Diabetic nerves (D) had significantly reduced NBF and resembled normal nerves (N) following NOLA. Fidarestat dose-dependently improved NBF (D+1, D+4). $* p<0.05, * * p<0.01$ 

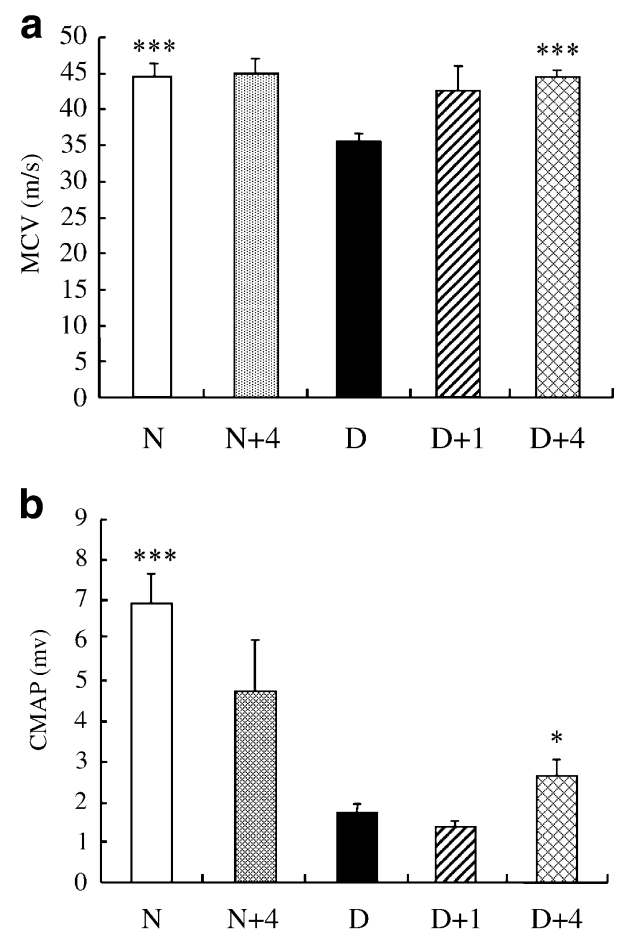

Fig. $2 \mathrm{MCV}$ (a) and CMAP (b) in sciatic-tibial nerve. ${ }^{*} p<0.05$, $* * * p<0.001$ vs D group

In vitro recordings

There were two major components of the monophasic compound action potential of the sciatic nerve in these rats. The conduction velocity of the fastest wave (A-wave) was $70.6 \pm 11.7 \mathrm{~m} / \mathrm{s}$ (mean $\pm \mathrm{SE}$ ) and the amplitude was $3.7 \pm$ $1.0 \mathrm{mV}$; the conduction velocity and amplitude of the second wave (C-wave) was $3.6 \pm 0.6 \mathrm{~m} / \mathrm{s}$ and $264 \pm 41.7 \mu \mathrm{V}$, respectively, in the $\mathrm{N}$ group.

In the $\mathrm{D}$ group, the conduction velocity and amplitude of the A-wave were decreased compared with those in the $\mathrm{N}$ group (Fig. 3), but the difference did not reach statistical significance. Among the groups of diabetic rats, fidarestat supplementation improved conduction velocity of the A-wave significantly ( $\mathrm{D}+1 p<0.001, \mathrm{D}+4 p<0.05)$ but did not increase amplitude.

Both conduction velocity and amplitude of the C-wave were significantly reduced in the $\mathrm{D}$ group (conduction velocity $p<0.001$, amplitude $p<0.01)$. In the $\mathrm{D}+4$ group, fidarestat significantly increased $\mathrm{C}$-wave amplitude $(p<0.05)$ and the increase in conduction velocity approached significance ( $p=0.07$ ) (Fig. 4).

Sorbitol, fructose and GSH

Sorbitol and fructose content in the D group increased 11fold and 5.5-fold compared with those in the untreated $\mathrm{N}$ group (Fig. 5). Low-dose fidarestat $(1 \mathrm{mg} / \mathrm{kg})$ partially
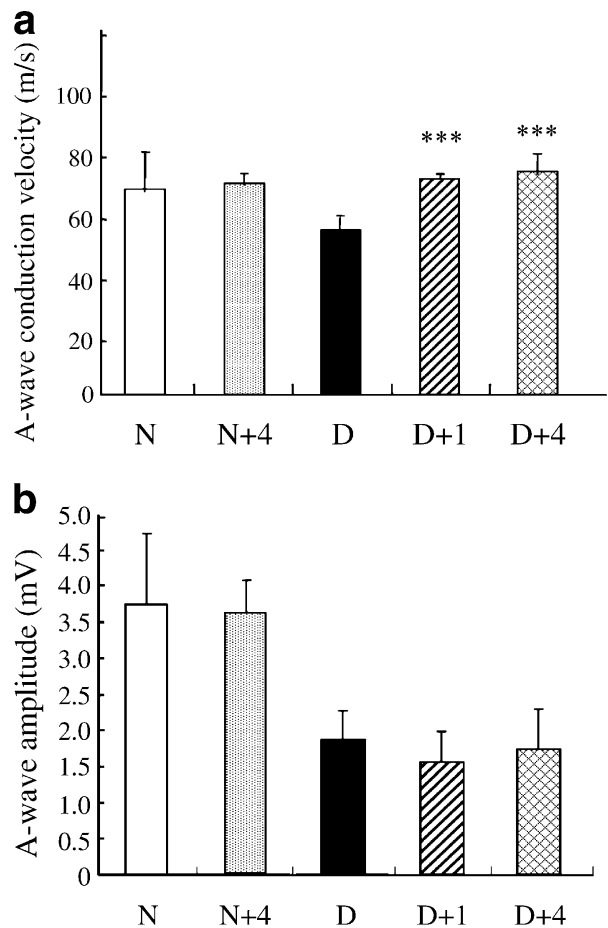

Fig. 3 Conduction velocity (a) and amplitude of A-wave (b) in in vitro recording. $* * * p<0.001$ vs $\mathrm{D}$ group

corrected this increase in sorbitol and fructose, while $4 \mathrm{mg} / \mathrm{kg}$ supplementation resulted in normalisation of both sorbitol and fructose (sorbitol $85-104 \%$ inhibition, fructose $59-95 \%$ inhibition). Sorbitol and fructose levels in

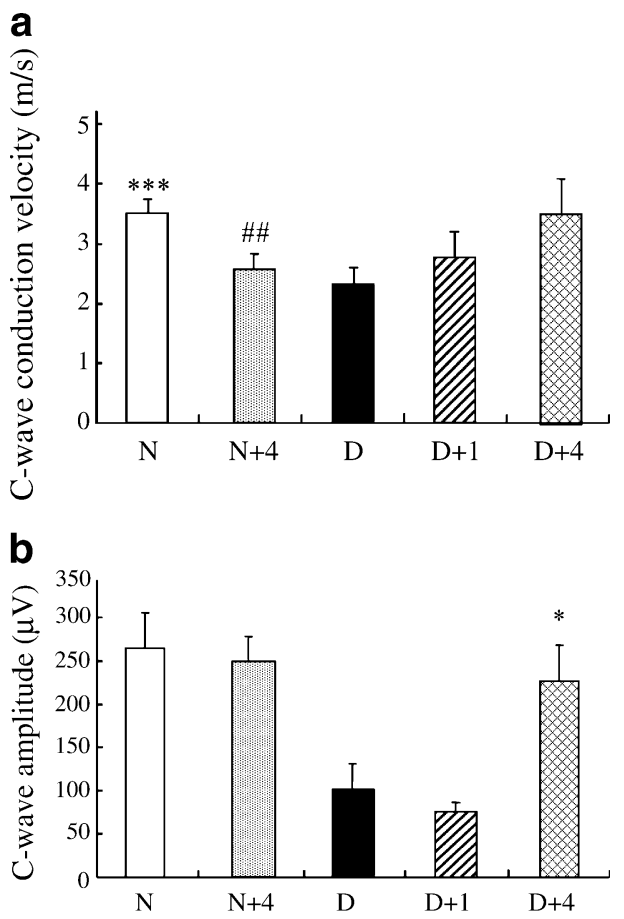

Fig. 4 Conduction velocity and the amplitude of $\mathrm{C}$-wave in chamber study. ${ }^{\# \#} p<0.01$ vs $\mathrm{N}$ group. ${ }^{* * *} p<0.001$ vs $\mathrm{D}$ group 

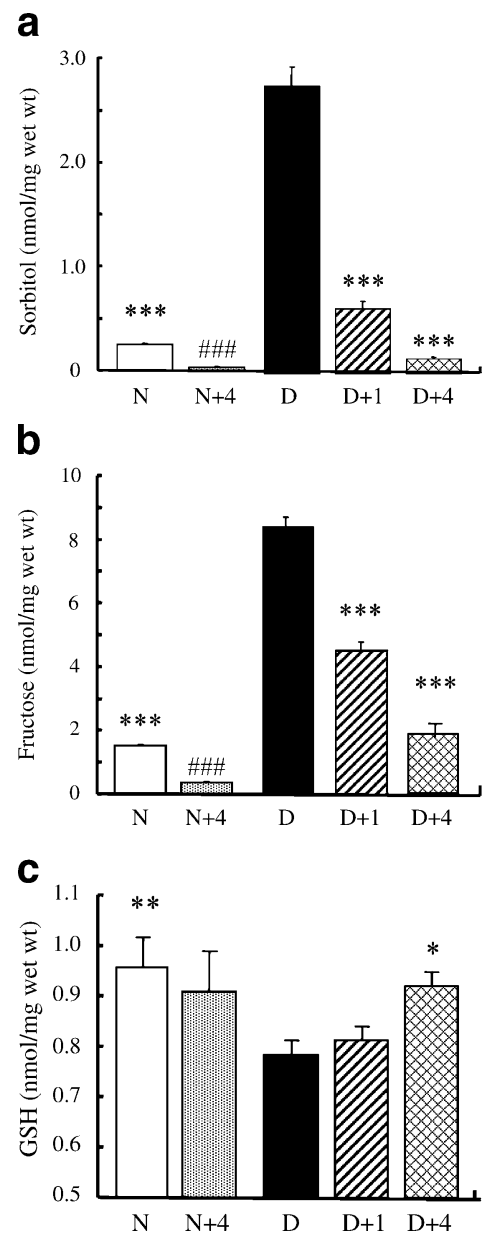

Fig. 5 Sorbitol (a), fructose (b) and GSH (c) contents in sciatic nerve.

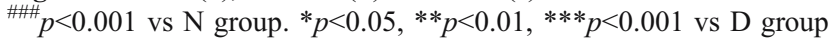

the fidarestat-treated normal group were lower than in the untreated normal group.

The GSH content in the untreated diabetic group was significantly reduced (Fig. 5). Fidarestat at the dose of $4 \mathrm{mg} / \mathrm{kg}$ corrected this decrement. There was no difference between the untreated normal group and the fidarestattreated normal group in GSH content.

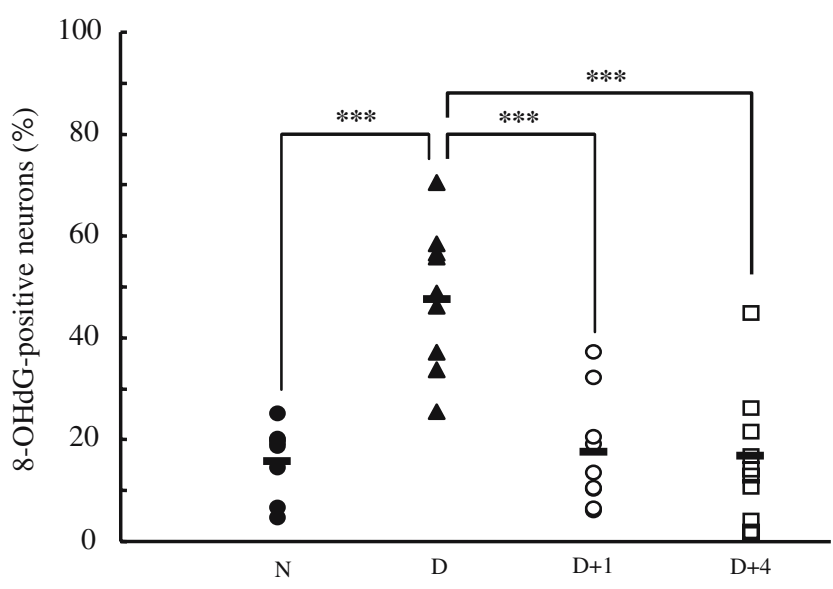

Fig. 7 The prevalence of 8-OHdG-positive neurons in the D group increased by three-fold compared with that in the $\mathrm{N}$ group. Fidarestat maintained the prevalence at the normal level at a dose of $1 \mathrm{mg} / \mathrm{kg}$. The horizontal line represents the mean value of each group

\section{8-OHdG}

The prevalence of 8-OHdG-positive neurons in the $\mathrm{D}$ group increased three-fold compared with that in the $\mathrm{N}$ group $(16.1 \%)$. Fidarestat at either dose normalised the prevalence of 8 -OHdG neurons ( $1 \mathrm{mg} / \mathrm{kg}: 17.3 \% ; 4 \mathrm{mg} / \mathrm{kg}: 16.9)$ (Figs 6, 7).

Diameter of DRG and ratio of small:medium:large DRG neurons

The diameter of DRG neurons in the D group decreased by $10 \%(p<0.05)$ compared with that in the $\mathrm{N}$ group. Fidarestat corrected this decrease in diameter by 40 and $70 \%$ at doses of 1 and $4 \mathrm{mg} / \mathrm{kg}$.

In the $\mathrm{D}$ group, the diameter distribution histogram was altered from that of the $\mathrm{N}$ and fidarestat-treated diabetic groups. The percentage of small diameter neurons was significantly increased, with a corresponding reduction in large diameter neurons, for the $\mathrm{D}$ group compared with the
Fig. 6 DRG size in the N (a) and $\mathrm{D}$ (b) groups. DRG size in the $\mathrm{D}$ group was reduced significantly compared with that in the $\mathrm{N}$ group

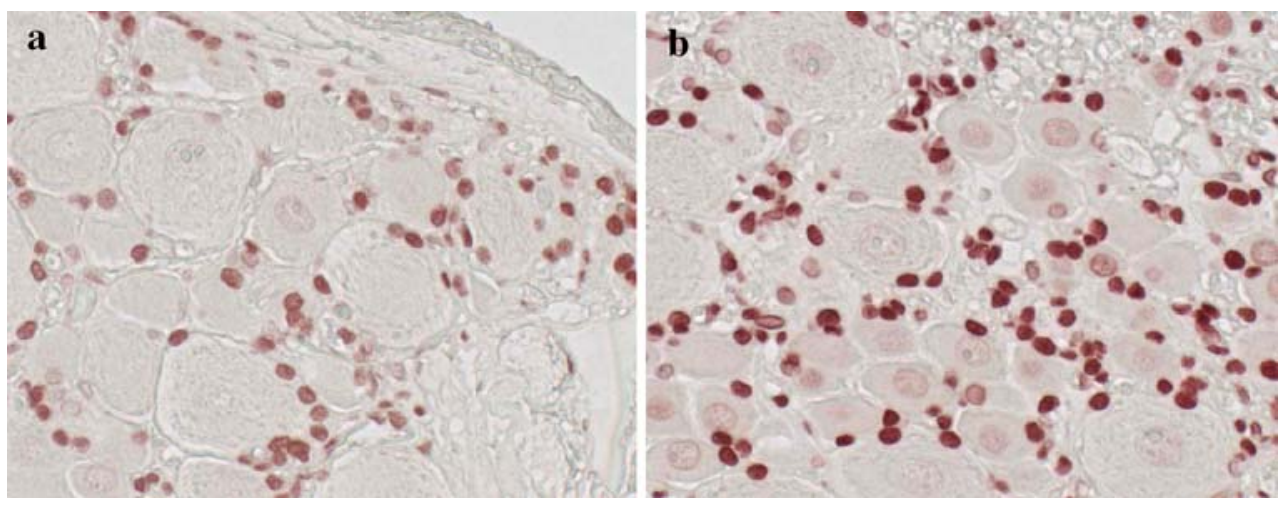




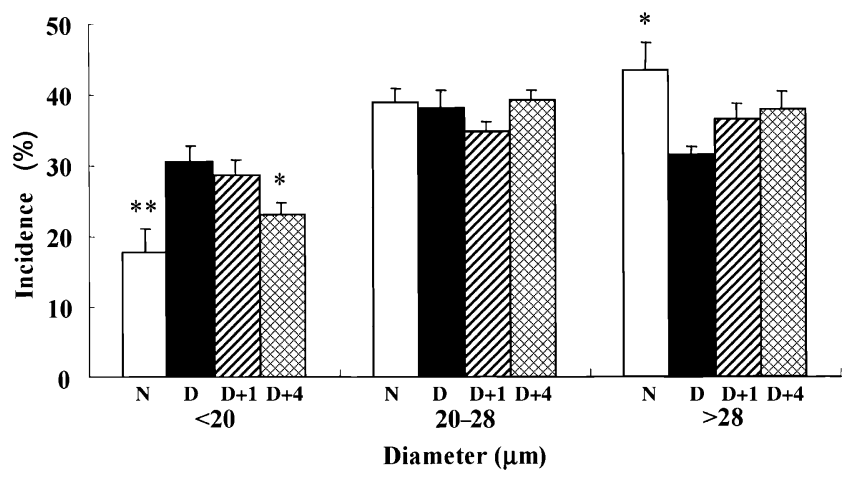

Fig. 8 Diameter histograms of experimental groups for all DRG neurons. ${ }^{*} p<0.05,{ }^{*} p<0.01$ vs $\mathrm{D}$ group

$\mathrm{N}$ group. Fidarestat corrected the increase and reduction in number of small and large neurons in a dose-dependent manner (Fig. 8).

\section{Discussion}

There are a number of key findings and some novel findings in this study. We confirm that EDN resulted in a significant reduction in NBF. We demonstrate, for the first time, with in vitro compound action potential recordings that unmyelinated nerve fibres are involved in addition to myelinated nerve fibres. Oral feeding of fidarestat improved both the perfusion and conduction deficits in diabetic rats. The NOS experiments demonstrate that the NBF deficit in diabetic rats is due to NO deficiency. Fidarestat corrects the accumulation of sorbitol and fructose, indicating that aldose reductase inhibition will correct this deficiency. Finally, fidarestat corrects the reduction in GSH and immunohistochemical indices of oxidative stress-related DNA damage. These changes are accompanied by normalisation morphometric alterations in both large and small DRG neurons in EDN.

We used the $\mathrm{H}_{2}$-polarographic technique to measure NBF because of its ability to separate nutritive (capillary) from capacitative (arteriovenous) flow and its ability to measure NBF repeatedly under various physiological conditions [8]. Other techniques, such as ${ }^{14} \mathrm{C}$-labelled iodoantipyrine require killing the animal for a single blood flow determination [21]. Laser Doppler velocimetry measures NBF dynamically but does not separate endoneural capillary flow from flow in larger vessels, and does not provide NBF measurements in absolute units [25].

The combination of microelectrode polarography and topical superfusion of test agents is particularly suited to the study of peripheral nerve vasoreactivity [5, 20]. Nerve microvasculature is much less vasoreactive than other tissues and responds to changes in blood pressure in a largely passive fashion, so that NBF alterations following the systemic administration of vasoactive agents might reflect changes in blood pressure rather than those of arteriolar tone [9]. The second advantage of our preparation is that the perineurial barrier, with its tight junction, isolates nerve epineurium from endoneural contents, permitting the study of nerve epineurial arterioles in relative isolation [26].

Our studies lend support to the concept that NBF reduction in EDN is due to a reduction in NO. Fidarestat increased sciatic NBF in diabetic rats but had no effect on normal rats. Furthermore, NOS inhibitors did not inhibit NBF in untreated EDN diabetic rats (presumably because NOS was already inhibited) and this deficit was corrected by fidarestat supplementation. These results suggest that the correction of NBF with fidarestat was due mainly to improved NOS.

The electrophysiological studies provide insights into the dose-dependency and fibre populations involved. In the D+1 group the CMAP was not significantly improved compared with the control group, and the improvement in conduction velocity did not reach statistical significance. A further increase in dose (D+4 group) resulted in significant improvement in both CMAP and the MCV. In vitro monophasic recordings enabled a separate evaluation of large myelinated and unmyelinated nerve fibres. The monophasic compound action potential recorded from peripheral nerve is comprised of three major components: A, B and C waves. These amplitudes form the basis of one classification of nerve fibre. Brinley showed that conduction velocities of $\mathrm{A}, \mathrm{B}$ and $\mathrm{C}$ waves in the cat were $15-120,3-15$ and $0.7-$ $2.3 \mathrm{~m} / \mathrm{s}$, respectively [27]. Hopkins and Lambert reported that amplitude of A fibre was $200-1600 \mu \mathrm{V}$ in sural nerve of rats [28]. We focused on the fastest and largest component (A wave) and the slowest component ( $\mathrm{C}$ wave), as indices of the largest myelinated and the unmyelinated fibres. Fidarestat therapy resulted in an improvement in velocity of large fibres and amplitude of small fibres significantly. This is the first report that EDN affects unmyelinated fibres and that fidarestat supplementation will improve this deficit.

The alterations in $\mathrm{C}$ fibres have therapeutic implications. Sweitzer et al reported that SD-292, a p38 mitogenactivated protein kinase (MAPK) inhibitor, prevented the exacerbation of $\mathrm{C}$-fibre-meditated thermal hyperalgesia in diabetic rats [29]. Price et al. [30] also reported that fidarestat will prevent activation of p38 MAPK and improve motor and sensory nerve conduction in EDN. These studies suggest a potential therapeutic value of $\mathrm{p} 38$ MAPK inhibitors in the treatment of aberrant pain sensitivity produced by diabetes. Fidarestat, by its action on p38 MAPK, could suppress the C-fibre-mediated aberrant pain sensitivity in diabetic neuropathy.

Our studies support an important role of oxidative stress. GSH is a reliable and sensitive index of ongoing oxidative stress in EDN [19], and reduction of NBF is one of the major mechanisms of free-radical generation in diabetes 
[19]. In our study, fidarestat improved NBF and normalised endoneurial GSH, supporting the concept that increased NBF by fidarestat reduced oxidative stress of nerve in diabetic rats. There is considerable evidence of hyperglycaemia-induced oxidative injury. Chronic EDN results in DRG neuronal apoptosis [23], with a significant reduction in large DRG neurons [24]. We now provide evidence that both the increase in 8-OHdG-positive neurons as well as the morphometric changes can be improved with fidarestat treatment. Of particular interest is the role of oxidative injury on C-fibre involvement, and function and the ability of fidarestat to prevent these alterations.

The present study was a comprehensive evaluation of the effects of fidarestat, combining physiological studies of perfusion, electrophysiological studies, in vivo and in vitro of large and small fibres separately, combined with biochemical, immunohistochemical and morphometric evaluation of oxidative injury. This study, together with previously published work, provides an integrated view of the interactions of myoinositol-polyphosphoinositide perturbations with oxidative stress and its sequelae. Hyperglycaemia results in excessive polyol pathway activity and a reduction in myoinositol and $\mathrm{Na}^{+} \mathrm{K}^{+}$-ATPase activity $[1,3]$ resulting in a reduction in NADPH. The reduction in $\mathrm{NADPH}$ results in a reduction in $\mathrm{NO}$ and a reduction in GSH (both NADPH-requiring). A major site of generation of free radicals is mitochondria [31]. Fidarestat is active in several sites of action. It is a powerful ARI and reduces polyol accumulation [32]. The other mechanism is the polyol pathway being active in the endothelial cell. Aldose reductase and NOS both require and compete for NADPH as a cofactor. Our studies with NOLA suggest that a second site of action in improvement of NOS [4-6]. A third site of action is at the DRG where is reduces oxidative damage, prevents the loss of large diameter neurons and corrects dysfunction of C-fibre.

However, without the demonstration that a therapeutic intervention can normalise the defective axonal regeneration characteristics of diabetes, then it cannot considered to be fully competent against neuropathy in this study.

Tomlison et al. found that ARI such as sorbitol or imirestat had little effect on endoneurial NO or NBF to normalise conduction velocity in diabetes rats [33]. Our data showed significant effects on both NO and NBF. A previous study reported that fidarestat had stronger aldose reductase inhibition and longer half-life in the peripheral nerve than other ARIs [32]. These points could explain why fidarestat is more effective on NO and NBF in improving conduction velocity. However, as Tomlison et al. described, some other mechanisms based on more direct effects on axons or Schwann cell functions may also be associated with the effect of ARI. More pathological and physiological experiments will be necessary to clarify these issues.
Acknowledgement The authors thank M. Okuyama for technical support in the measurement of 8-OHdG.

Duality of interest There is no duality of interest in this study.

\section{References}

1. Greene DA, Sima AA, Stevens MJ et al (1993) Aldose reductase inhibitors: an approach to the treatment of diabetic nerve damage. Diabetes Metab Rev 9:189-217

2. Greene DA, Yagihashi S, Lattimer SA, Sima AA (1998) Nerve $\mathrm{Na}, \mathrm{K}-\mathrm{ATPase}$, conduction and myo-inositol in the insulin-deficient BB rat. Am J Physiol 247:E534-E539

3. Hohman TC, Kwon HM (1995) Two separate mechanisms for the glucose inhibition of myo-inositol transport. In: Hotta N, Greene DA, Ward JD, Sima AA, Boulton AJ (eds) Diabetic neuropathy: new concepts and insights. Elsevier, Amsterdam, pp 43-58

4. Raccah D, Coste T, Cameron NE, Dyfayet D, Vague P, Hohman TC (1998) Effect of the aldose reductase inhibitor torestat on nerve conduction velocity, $\mathrm{Na} / \mathrm{K}$ ATPase activity, and polyols in red blood cells, sciatic nerve, kidney cortex, and kidney medulla of diabetic rats. J Diab Complicat 12:154-162

5. Kihara M, Low PA (1995) Impaired vasoreactivity to nitric oxide in experimental diabetic neuropathy. Exp Neurol 132:180-185

6. Nagamatsu M, Nicklander KK, Schmelze J, Low PA (1995) Lipoic acid improves nerve blood flow, reduces oxidative stress and improves distal nerve conduction in experimental diabetic neuropathy. Diab Care 18:1160-1167

7. Cameron NE, Cotter MA (1994) The relationship of vascular changes to metabolic factors in diabetes mellitus and their role in the development of peripheral nerve complications. Diab Metab Rev 10:189-224

8. Low PA, Tuck R (1984) Effects of changes of blood pressure, respiratory acidosis and blood flow in the sciatic nerve of the rat. J Physiol 347:513-524

9. Low PA, Lagerlund TD, McManis PG (1989) Nerve blood flow and oxygen delivery in normal, diabetic and ischemic neuropathy. Int Rev Neurobiol 31:355-426

10. Kihara M, Mitsui MK, Mitsui Y et al (1999) Altered vasoreactivity to angiotensin II in experimental diabetic neuropathy: role of nitric oxide. Muscle Nerve 22:920-925

11. Brownlee M (2004) The pathobiology of diabetic complications: a unifying mechanism. Diabetes 54:1615-1625

12. Feldman EL (2003) Oxidative stress and diabetic neuropathy: a new understanding of an old problem. J Clin Invest 111: 431-433

13. Obrosova IG, Van Huysen C, Fathallah L, Cao XC, Green DA, Stevens MJ (2002) An aldose reductase inhibitor reverses early diabetes-induced changes in peripheral nerve function, metabolism, and antioxidative defence. FASEB J 16:123-125

14. Uehara K, Yamagishi S, Otsuki S, Chin S, Yagihashi S (2004) Effects of polyol pathway hyperactivity on protein kinase $\mathrm{C}$ activity, nociceptive peptide expression, and neuronal structure in dorsal root ganglia in diabetic mice. Diabetes 53:3239-3247

15. Obrosova IG, Minchenko AG, Vasupuram R et al (2003) Aldose reductase inhibitor fidarestat prevents retinal oxidative stress and vascular endothelial growth factor overexpression in streptozotocin-diabetic rats. Diabetes 52:864-871

16. Obrosova IG, Pacher P, Szabo C et al (2005) Aldose reductase inhibition counteracts oxidative-nitrosative stress and poly(ADP- 
ribose) polymerase activation in tissue sites for diabetes complications. Diabetes 54:234-242

17. Kato N, Mizuno K, Makino M, Suzuki T, Yagihashi S (2000) Effects of 15-month aldose reductase inhibition with fidarestat on the experimental diabetic neuropathy in rats. Diabetes Res Clin Pract 50:77-85

18. Dandona P, Thusu K, Cook S et al (1996) Oxidative damage to DNA in diabetes mellitus. Lancet 17:444-445

19. Low PA, Nickander KK, Trischler HJ (1997) The roles of oxidative stress antioxidant treatment in experimental diabetic neuropathy. Diabetes 46:S38-S42

20. Sugimoto H, Monafo WW, Eliasson SG (1986) Regional sciatic and muscle blood flow in conscious and anesthetized rats. Am J Physiol 251:H1211-H1216

21. Gurrant GO, Moss CW (1984) Determinations of monosaccharide as aldononitrite, o-methyleoxime, alditol, and cyclitol acetate derivatives by gas chromatography. Anal Chem 56:633-638

22. Takahashi S, Hirose M, Tamano S et al (1998) Immunohistochemical detection of 8-hydroxy-2'-deoxyguanosine in paraffinembedded sections of rat liver after carbon tetrachloride treatment. Toxicol Pathol 26:247-252

23. Schmeichel AM, Schmeizer JD, Low PA (2003) Oxidative injury and apoptosis of dorsal root ganglion neurons in chronic experimental diabetic neuropathy. Diabetes 52:165-171

24. Kishi M, Tanabe J, Schmelzer JD, Low P (2002) Morphometry of dorsal root ganglion in chronic experimental diabetic neuropathy. Diabetes 51:819-824

25. Takeuchi M, Low PA (1987) Dynamic peripheral nerve metabolic and vascular responses to exsanguination. Am J Physiol 253:E349-E353
26. Olsson Y, Reese TS (1971) Permeability of vasa nervosum and perineurium in mouse sciatic nerve studied by fluorescence and electron microscopy. J Neuropathol Exp Neurol 30: $105-119$

27. Brinley FJ Jr (1980) Excitation and conduction in nerve fibers. In: Mountcastle VB (ed) Medical Physiology. The C.V. Mosby Company, St Louis, pp 46-81

28. Hopkins AP, Lambert EH (1972) Conduction in unmyelinated fibers in experimental neuropathy. J Neurol Neurosurg Psychiatry $35: 163-169$

29. Sweitzer SM, Medicherla S, Almirez R (2004) Antinociceptive action of p38 alpha MAPK inhibitor, SD-282, in a diabetic neuropathy model. Pain 109:409-419

30. Price SA, Agthong S, Middlemas AB, Tomlinson DR (2004) Mitogen-activated protein kinase p38 mediates reduced nerve conduction velocity in experimental diabetic neuropathy: interactions with aldose reductase. Diabetes 53:1851-1856

31. Feldman EL, Russell JW, Sullivan KA, Golovoy D (1999) New insights into the pathogenesis of diabetic neuropathy. Curr Opin Neurol 12:533-563

32. Hotta N, Toyota T, Matsuoka K et al (2001) Clinical efficacy of fidarestat, a novel aldose reductase inhibitor, for diabetic peripheral neuropathy: a 52-week multicenter placebo-control double-blind parallel group study. Diab Care 24:1776-1782

33. Tomlison DR, Dewhurst M, Stevens EJ, Omawari N, Carrington AL, Vo PA (1998) Reduced nerve blood flow in diabetic rats: relationship to nitric oxide production and inhibition of aldose reductase. Diabet Med 15:579-585 